\title{
Scattering from sidewall deformations in photonic crystals
}

\author{
Christopher G. Poulton, Manfred Müller, and Wolfgang Freude \\ Institute of High-Frequency and Quantum Electronics, University of Karlsruhe, Karlsruhe, Germany
}

Received July 6, 2004; revised manuscript received December 13, 2004; accepted January 10, 2005

\begin{abstract}
We present results from a new method for the solution of electromagnetic scattering from finite sets of twodimensional cylinders exhibiting sidewall deformations. Our model treats the sidewall deviation as a small perturbation on a circle; the response of a single cylinder to an arbitrary external field can then be calculated, and the interactions between the cylinders can be computed with a multipole method. This leads to a series of model problems for successively higher asymptotic orders. Because most of the computation time is taken up with solving the original, unperturbed problem, a large number of additional types of perturbation can be examined at little extra numerical cost. (C) 2005 Optical Society of America
\end{abstract}

OCIS codes: $250.5300,230.7370,230.3120,290.5880$.

\section{INTRODUCTION}

The realization of photonic crystals as practical devices has reached a critical stage in the last year or so. Many groups have reported large losses, in the order of $20 \mathrm{~dB} / \mathrm{cm},{ }^{1,2}$ for line-defect waveguides. Because attenuation in annealed waveguides has been measured to be considerably lower, ${ }^{3}$ these losses often are attributed to sidewall roughness that occurs owing to inaccuracies in the fabrication process. Technologies such as E-beam lithography, ion-beam milling, and reactive ion etching using masks leave variations both in the positions of the photonic-crystal elements and in the smoothness of their sidewalls of up to $30 \mathrm{~nm}$. For a slab photonic crystal with a period of $0.5 \mu \mathrm{m}$ and a cylinder radius of $150 \mathrm{~nm}$, this represents a significant deviation from the ideal case, and it is important to know whether functioning devices can be made from such structures.

For the study of the phenomena of sidewall deviation in photonic crystals it is necessary to have a method that is simultaneously accurate on a large scale (in order to incorporate the photonic-crystal effects) and that possesses the finesse required to resolve individual sidewall features. Among the candidates for these are adaptive grid finite-element methods ${ }^{4}$ and (promisingly) wavelet expansion methods. ${ }^{5}$ In both of these methods the computation time and memory load are increased dramatically with increased resolution of the boundary perturbation. We introduce here a method that is able to compute very large problems with a high short-range resolution, through combination of two standard approaches: that of asymptotic theory ${ }^{6-8}$ and of the multipole method. ${ }^{9}$ If one assumes that the unperturbed problem is readily solved, then we find that one can split the problem into two parts; asymptotics are used for calculation of the response of each cylinder to an arbitrary external field, and then the multipole method is used for computation of the effect of the interaction between the cylinders themselves. This leads to a series of model problems, the results of which can be summed to obtain the full solution. Most interest- ingly, the computation of the perturbed structure requires a comparatively small amount of extra computation, so a large number of perturbational configurations can be studied at little extra computational cost, provided that the original unperturbed problem can be solved.

The problem of scattering from perturbed cylinders has been studied previously, particularly in the context of random disorder in the size and position of photonic-crystal elements. A popular technique for the investigation of the effects of fabrication-related disorder on photonic-crystal band structure is to use a plane-wave expansion combined with a supercell approach. ${ }^{10-13}$ In this method the disordered system is approximated as a periodic system with a period large enough to guarantee negligible coupling between neighboring supercells. With this method, it was found that for a three-dimensional structure consisting of vertical air holes in a vertically periodic highindex ( $\mathrm{Si}$ or $\mathrm{GaAs}$ ) arrangement with horizontal air bars, the size of the bandgap is tolerant to significant amounts of deviation from a perfect structure. ${ }^{10}$ For a woodpile structure, it was found ${ }^{11}$ that disorder in the position of the elements had the greatest effect on the transmission; however this too was relatively small. The situation appears to be different for photonic crystals consisting of air spheres in high-contrast background material. In the presence of disorder in the positions and the radii of the spheres it was observed that the bandgap closes at a fluctuation magnitude as small as $2 \%$ of the lattice constant. $^{12}$

The effects of disorder have also been studied for the more traditional structures consisting of two-dimensional (2D) circular holes or inclusions in high-contrast background material. For a 2D hexagonal photonic crystal consisting of disordered air cylinders in a high-index background, the transmission was calculated ${ }^{14}$ by use of a combination of the transfer matrix method and the multiple-scattering technique. In this study the authors were able to investigate the effect of radial disorder on the transmission spectra for a range of different realizations. 
Asatryan et $a .^{15}$ used a multipole method to calculate transmission through a layered stack of cylinders that exhibited radial disorder and were able to derive a semianalytic formula for the localization length. In another study, a perturbation technique was developed ${ }^{12}$ in order to speed up the lengthy computation process based on the plane-wave supercell approach. The authors observed that, for disordered 2D photonic crystals consisting of high-index cylinders in air, the size of the first and second bandgaps was not very much affected by random positional disorder even as large as half the cylinder radius. On the other hand, it was observed that a random variation in cylinder radius of $33 \%$ reduced the size of the band gaps by a factor of one half.

Other authors have studied the problem of scattering from cylinders that are perturbed in shape. Wait ${ }^{6}$ used a perturbation method for study of the effects of scattering from cylinders with irregular boundaries. Agmon et al. ${ }^{7}$ derived effective boundary conditions for problems involving general elliptical partial differential equations. In another, related study, ${ }^{16}$ both perturbation methods and finite-element analysis were used for calculation the dispersion properties of a deformed photonic crystal. The boundary integral equation method has also been adapted $^{17}$ for investigation of scattering from arrays of noncircular cylinders. Tanaka et $a l{ }^{18}$ used a finitedifference time-domain (FDTD) method to examine transmission through a photonic-crystal line defect whose elements remained circular yet had a conical variation along their axes. At this time, however, there has been no systematic study of the effects of random perturbations of shape on the transmission properties of photonic crystals.

Of all of the studies mentioned, only two ${ }^{14,15}$ were able to study an ensemble of realizations and thereby analyze the statistics of the bandgaps. There have also been few investigations of the effect of disorder on line-defect waveguides (an exception being that of Tanaka ${ }^{18}$ ), and those that exist study only single realizations owing to the computational expense. For such analysis, it is necessary to employ a method that can calculate the transmission properties, with respect to both shape and positional disorder, of many systems quickly and efficiently. We present here an efficient algorithm for research in this direction.

\section{FORMULATION OF THE PROBLEM}

We consider an electromagnetic wave that strikes a set of infinitely extended cylinders placed perpendicular to the $(x, y)$ plane (see Fig. 1). Each cylinder can represent either a dielectric inclusion or a void within a dielectric matrix and possesses a $z$-constant noncircular cross section. We assume that the wave strikes the cylinders perpendicularly. In this case the problem can be entirely formulated in terms of the $z$ component of the electric field (in the case of TM polarization) or of the magnetic field (in the case of TE polarization). Denoting the refractive index of the matrix material as $n_{1}$ and that of the cylinders themselves as $n_{2}$, we assume a time dependence ansatz $\exp$ ( $-i \omega t$ ) and find that both TE and TM fields satisfy the Helmholtz equation

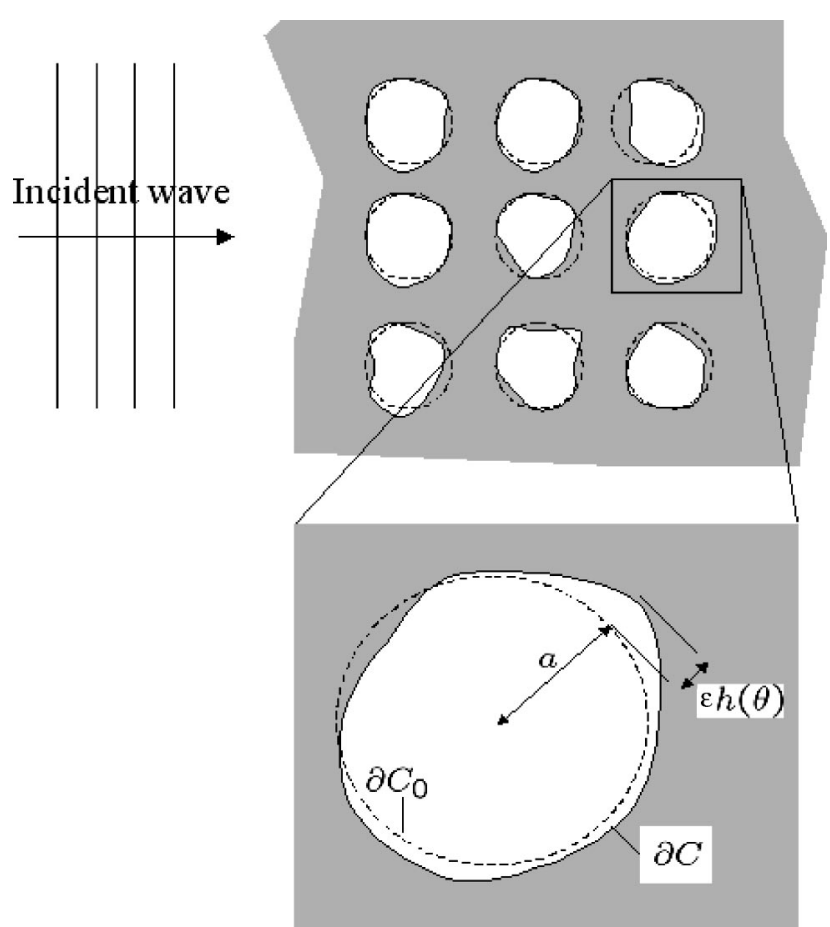

Fig. 1. Geometry in the $(x, y)$ plane. The cylinders are either dielectric inclusions or voids in an infinitely extended dielectric matrix and have a cross section that is constant in the $z$ (intothe-plane) direction.

$$
\left(\frac{\partial^{2}}{\partial x^{2}}+\frac{\partial^{2}}{\partial y^{2}}+k^{2}\right) u^{e}(x, y)=0
$$

in the region exterior to the cylinders, and

$$
\left(\frac{\partial^{2}}{\partial x^{2}}+\frac{\partial^{2}}{\partial y^{2}}+n_{c}^{2} k^{2}\right) u^{i}(x, y)=0
$$

in the interior region, where $k=n_{1} \omega / c$ is the perpendicular wave number in the homogeneous matrix material and $n_{c}=n_{2} / n_{1}$ is the relative refractive index of the cylinders. If we divide the total field into the incident wave $\left(u_{\text {inc }}\right)$ and the response $\left(u_{\text {resp }}\right)$, then the latter field must satisfy the Sommerfeld radiation condition for outgoing waves in two dimensions.

If the sidewall deformation is not large, the boundary $\partial C$ of each cylinder can be written as a perturbation on a circle, thus

$$
\partial C=\{(r, \theta): r=a+\epsilon h(\theta)\} .
$$

We denote the unperturbed boundary by $\partial C_{0}$. Here $a$ is the radius that can be inscribed in all cylinders of the set, $h(\theta)$ is a smooth function, and $\epsilon$ is a small parameter with the dimension of length. The cylinders are therefore convex and are "smooth" in the sense that they possess no sharp jumps or kinks on their boundaries. The function $h(\theta)$ we normalize such that

$$
\frac{1}{2 \pi} \int_{0}^{2 \pi}|h(\theta)|^{2} \mathrm{~d} \theta=1
$$

so that $\epsilon$ becomes the rms deviation from the unperturbed boundary. The subsequent analysis assumes that $\epsilon$ is 
small in comparison with the size of the cylinders and is typically not larger than one tenth of the mean radius.

The total field will satisfy the appropriate boundary conditions arising from the continuity of tangential components of the electric and magnetic fields on the boundary. For the purposes of illustration we will concentrate on the TE polarization; the TM case differs only in a couple of details. The conditions for the field $u(x, y)$ on the boundary $\partial C$ are then

$$
\left.u^{e}\right|_{\partial C}-\left.u^{i}\right|_{\partial C}=0
$$

and

$$
\left.\frac{\partial u^{e}}{\partial n}\right|_{\partial C}-\left.\frac{1}{n_{c}^{2}} \frac{\partial u^{i}}{\partial n}\right|_{\partial C}=0
$$

where the derivative $\partial / \partial n$ signifies the outward normal derivative on the cylinder boundary.

\section{ASYMPTOTIC PERTURBATION OF THE BOUNDARY CONDITIONS}

We now proceed to the asymptotic treatment of the case where the parameter $\epsilon$ in Eq. (3) is small. By doing so we hope to reduce the full problem with a deformed boundary to a series of model problems that are in some sense easier to solve.

We first expand the solution around a given cylinder in powers of $\epsilon$ :

$$
u(r, \theta)=\sum_{j=0}^{\infty} u_{j}(r, \theta) \epsilon^{j}
$$

where $(r, \theta)$ are polar coordinates centered on the cylinder. The zeroth order $(j=0)$ solution $u_{0}$ then corresponds to the solution from a set of unperturbed (i.e., circular) cylinders of radius $a$. The mathematical justification for such an expansion comes from the theory of asymptotic estimates for the solution of elliptic partial differential equations, as developed by Agmon et al. ${ }^{7}$ and other authors. 8

We note that the Helmholtz equation is satisfied by each "subfield" $u_{j}$. Namely, we specify that

$$
\left(\frac{\partial^{2}}{\partial x^{2}}+\frac{\partial^{2}}{\partial y^{2}}+k^{2}\right) u_{j}^{e}(x, y)=0, \quad x^{2}+y^{2} \geqslant a^{2} .
$$

and

$$
\left(\frac{\partial^{2}}{\partial x^{2}}+\frac{\partial^{2}}{\partial y^{2}}+n_{c}^{2} k^{2}\right) u_{j}^{i}(x, y)=0, \quad x^{2}+y^{2}<a^{2} .
$$

The connection between the internal and external fields for each order can be deduced from the boundary conditions [Eqs. (5) and (6)] on the perturbed boundary. First we examine the boundary condition in Eq. (5), which expresses continuity of the $z$ component of the electric or magnetic field across the perturbed boundary. With Taylor's series the fields $u^{e}$ and $u^{i}$ on the perturbed boundary can be expanded about $r=a$ so that

$$
\left.u^{e}\right|_{\partial C}-\left.u^{i}\right|_{\partial C}=\sum_{n=0}^{\infty} \frac{(\epsilon h)^{n}}{n !}\left(\frac{\partial^{n} u^{e}}{\partial r^{n}}-\frac{\partial^{n} u^{i}}{\partial r^{n}}\right)_{\partial C_{0}}
$$

where the subscript $\partial C_{0}$ indicates that the bracketed expression is to be evaluated on the unperturbed boundary. Substituting the ansatz [Eq. (7)] we obtain

$$
\begin{aligned}
\left.u^{e}\right|_{\partial C}-\left.u^{i}\right|_{\partial C} & =\sum_{n=0}^{\infty} \frac{[\epsilon h(\theta)]^{n}}{n !} \frac{\partial^{n}}{\partial r^{n}} \sum_{j=0}^{\infty} \epsilon^{j}\left(u_{j}^{e}-u_{j}^{i}\right)_{\partial C_{0}} \\
& =\sum_{n=0}^{\infty} \sum_{j=n}^{\infty} \epsilon^{j} \frac{[h(\theta)]^{n}}{n !}\left(\frac{\partial^{n} u_{j-n}^{e}}{\partial r^{n}}-\frac{\partial^{n} u_{j-n}^{i}}{\partial r^{n}}\right)_{\partial C_{0}},
\end{aligned}
$$

where we have rearranged the sums in the second step.

According to the boundary condition in Eq. (5) the total jump in field values across the boundary must be zero, so

$$
0=\sum_{n=0}^{\infty} \sum_{j=n}^{\infty} \epsilon^{j} \frac{[h(\theta)]^{n}}{n !}\left(\frac{\partial^{n} u_{j-n}^{e}}{\partial r^{n}}-\frac{\partial^{n} u_{j-n}^{i}}{\partial r^{n}}\right)_{\partial C_{0}} .
$$

Equating powers of $\epsilon$ and separating out the $n=0$ term, we obtain

$$
\left.u_{j}^{e}\right|_{\partial C_{0}}-\left.u_{j}^{i}\right|_{\partial C_{0}}=-\sum_{n=1}^{j} \frac{h^{n}(\theta)}{n !}\left(\frac{\partial^{n} u_{j-n}^{e}}{\partial r^{n}}-\frac{\partial^{n} u_{j-n}^{i}}{\partial r^{n}}\right)_{\partial C_{0}} .
$$

Thus we obtain a series of boundary conditions for successive problems in different orders of $\epsilon$, expressing a different jump in the field values for each order. That is,

$$
\begin{gathered}
\left.u_{0}^{e}\right|_{\partial C_{0}}-\left.u_{0}^{i}\right|_{\partial C_{0}}=0, \\
\left.u_{1}^{e}\right|_{\partial C_{0}}-\left.u_{1}^{i}\right|_{\partial C_{0}}=-\left(\frac{\partial u_{0}^{e}}{\partial r}-\frac{\partial u_{0}^{i}}{\partial r}\right)_{\partial C_{0}}, \\
\vdots \\
\left.u_{j}^{e}\right|_{\partial C_{0}}-\left.u_{j}^{i}\right|_{\partial C_{0}}=f_{j}(\theta),
\end{gathered}
$$

where

$$
f_{j}(\theta)=-\sum_{n=1}^{j} \frac{h^{n}(\theta)}{n !}\left(\frac{\partial^{n} u_{j-n}^{e}}{\partial r^{n}}-\frac{\partial^{n} u_{j-n}^{i}}{\partial r^{n}}\right)_{\partial C_{0}} .
$$

The effect of the boundary condition for the curved boundary $\partial C$ is thus replicated by a distribution of sources on the surface of the circular cylinder $\partial C_{0}$.

The same analysis, with a few modifications, holds for the condition on the field derivatives in Eq. (6). First, however, we need a representation of the derivative on the perturbed boundary $\partial / \partial n$ in polar coordinates. We note that the outward-pointing unit normal vector $\hat{\mathbf{n}}$ on the perturbed boundary can be written in polar coordinates as 


$$
\hat{\mathbf{n}}=\frac{(a+\epsilon h) \hat{\mathbf{r}}-\epsilon h^{\prime} \hat{\theta}}{\left[(a+\epsilon h)^{2}+\left(\epsilon h^{\prime}\right)^{2}\right]^{1 / 2}},
$$

where $\hat{\mathbf{r}}$ and $\hat{\theta}$ are the usual unit vectors in polar coordinates. Here we have dropped the dependency on $\theta$ for convenience and denoted $h^{\prime} \equiv \mathrm{d} h / \mathrm{d} \theta$. The normal derivative of any field on the boundary is then

$$
\begin{aligned}
\left.\frac{\partial u}{\partial n}\right|_{\partial C} & =[\hat{\mathbf{n}} \cdot \nabla u]_{\partial C} \\
& =\frac{(a+\epsilon h) \hat{\mathbf{r}}-\epsilon h^{\prime} \hat{\theta}}{\left[(a+\epsilon h)^{2}+\left(\epsilon h^{\prime}\right)^{2}\right]^{1 / 2}} \cdot\left(\frac{\partial u}{\partial r} \hat{\mathbf{r}}+\frac{1}{a+\epsilon h} \frac{\partial u}{\partial \theta} \hat{\theta}\right)_{\partial C} \\
& =\frac{1}{\mathcal{N}}\left[\left.(a+\epsilon h)^{2} \frac{\partial u}{\partial r}\right|_{\partial C}-\left.\epsilon h^{\prime} \frac{\partial u}{\partial \theta}\right|_{\partial C}\right],
\end{aligned}
$$

where $\mathcal{N}(\epsilon, \theta)=(a+\epsilon h)\left\{[a+\epsilon h(\theta)]^{2}+\left[\epsilon h^{\prime}(\theta)\right]^{2}\right\}^{1 / 2}$. The boundary condition in Eq. (6) can then be written

$$
\begin{aligned}
0= & \left(a^{2}+2 \epsilon a h+\epsilon^{2} h^{2}\right)\left(\frac{\partial u^{e}}{\partial r}-\frac{1}{n_{c}^{2}} \frac{\partial u^{i}}{\partial r}\right)_{\partial C} \\
& -\epsilon h^{\prime}\left(\frac{\partial u^{e}}{\partial \theta}-\frac{1}{n_{c}^{2}} \frac{\partial u^{i}}{\partial \theta}\right)_{\partial C} \cdot
\end{aligned}
$$

We now follow the steps given previously in Eqs. (7)-(13), substituting the Taylor series [Eq. (10)] as well as the asymptotic ansatz [Eq. (7)] into Eq. (20). The result, after the powers of $\epsilon$ are equated and the first term is separated out, is a little cumbersome to derive; however, the analogy with Eqs. (10)-(17) holds. This time, however, we obtain a jump in the normal derivative of the boundary conditions for each order in $\epsilon$, written in compact form as

$$
\left(\frac{\partial u_{j}^{e}}{\partial r}-\frac{1}{n_{c}^{2}} \frac{\partial u_{j}^{i}}{\partial r}\right)_{\partial C_{0}}=g_{j}(\theta)
$$

where

$$
\begin{aligned}
g_{j}(\theta)= & -\sum_{n=1}^{j} \frac{h^{n}}{n !}\left[\left(\frac{\partial^{n+1}}{\partial r^{n+1}}+\frac{2 n}{a} \frac{\partial^{n}}{\partial r^{n}}+\frac{n(n-1)}{a^{2}} \frac{\partial^{n-1}}{\partial r^{n-1}}\right.\right. \\
& \left.\left.-\frac{n h^{\prime}}{h a^{2}} \frac{\partial^{n}}{\partial r^{n-1} \partial \theta}\right)\left(u_{j-n}^{e}-\frac{1}{n_{c}^{2}} u_{j-n}^{i}\right)\right]_{\partial C_{0}} .
\end{aligned}
$$

Once again we obtain a series of boundary conditions that depend only on solutions of lower-order problems in $\epsilon$. The jump in the derivatives has the same effect as that of a distribution of surface currents on the unperturbed cylinder.

The total problem is now reduced to a series of linked subproblems, one for each field $u_{j}$, that satisfy the Helmholtz equation inside and outside the unperturbed cylinder and with boundary conditions that mimic the presence of surface charges and currents on the cylinder's surface. These "fictitious sources" depend only on the fields of lower asymptotic order and reconstruct the boundary conditions in the full solution.

\section{SOLUTION OF THE PROBLEM WITH MULTIPOLE SERIES}

We have reduced the scattering problem involving the perturbed boundary to a series of model problems involving the Helmholtz equation and linked boundary conditions. We now need a suitable method for solution of this series of problems. We choose to expand the field in polar coordinates with the origin located at the center of each unperturbed cylinder so that the field around cylinder $P$ is

$$
u_{j}^{e}(r, \theta)=\sum_{m=-\infty}^{\infty}\left[A_{m}^{j P} J_{m}(k r)+B_{m}^{j P} H_{m}(k r)\right] \exp (i m \theta)
$$

outside the perturbed boundary and

$$
u_{j}^{i}(r, \theta)=\sum_{m=-\infty}^{\infty} C_{m}^{j P} J_{m}\left(n_{c} k r\right) \exp (i m \theta)
$$

within the cylinder itself. Here the terms $J_{m}$ are Bessel functions of the first kind and $H_{m}$ are Hankel functions of the first kind, which represent outgoing waves according to our time-dependence ansatz. The superscript $P$ in the multipole coefficients indicates that the expansion is valid only in the vicinity of the Pth cylinder; in fact, it can be shown that the radius of convergence extends until the boundary of the nearest neighboring cylinder. ${ }^{19}$

There are several advantages for choosing such a series expansion. First, there is a clear distinction between the outgoing field from each cylinder [represented by the singular terms in the expansion in Eq. (23)] and the field that comes from all of the sources external to the cylinder (represented by the regular terms). The total response field is then a sum of Hankel functions, and so the Sommerfeld radiation condition is automatically satisfied. Second, such a series expansion can be shown to converge very rapidly. We will return to this point in Section 6 .

In terms of the multipole expansion [Eq. (23)], the series of problems reduces to a particularly convenient form. First we note that we can write the "boundary functions" $f_{j}(\theta)$ and $g_{j}(\theta)$ in a Fourier series:

$$
f_{j}(\theta)=\sum_{m=-\infty}^{\infty} f_{m}^{(j)} \exp (i m \theta), \quad g_{j}(\theta)=\sum_{m=-\infty}^{\infty} g_{m}^{(j)} \exp (i m \theta) .
$$

The coefficients $f_{m}^{(j)}$ and $g_{m}^{(j)}$ depend only on the multipole coefficients $A_{\ell}^{j P}, B_{\ell}^{J P}, C_{\ell}^{j P}$ of lower order in $\epsilon$, i.e., on terms of order j-1 or lower. To see this explicitly we first define the Fourier coefficients $h_{m}^{(n)}$ of the $n$th power of the perturbation by

$$
h^{n}(\theta)=\sum_{m=-\infty}^{\infty} h_{m}^{(n)} \exp (\operatorname{im} \theta)
$$

We then substitute this, together with the multipole expansions of Eqs. (23) and (24), into Eqs. (17) and (22). This results in explicit expressions for the Fourier coefficients of the "boundary functions:" 


$$
\begin{aligned}
f_{m}^{(j)}= & -\sum_{n=1}^{j} \sum_{\ell=-\infty}^{\infty} \frac{k^{n} h_{m-\ell}^{(n)}}{n !}\left[A_{\ell}^{j-n, P} J_{\ell}^{(n)}(k a)+B_{\ell}^{j-n, P} H_{\ell}^{(n)}(k a)\right. \\
& \left.-n_{c}^{n} C_{\ell}^{j-n, P} J_{\ell}^{(n)}\left(n_{c} k a\right)\right], \\
g_{m}^{(j)}= & \sum_{n=1}^{j} \sum_{\ell=-\infty}^{\infty} \frac{h_{m-\ell}^{(n)} k^{n+1}}{n !}\left\{\left[J_{\ell}^{(n+1)}(k a)+\frac{2}{k a} J_{\ell}^{(n)}(k a)\right.\right. \\
& \left.+\frac{n+n(n-1) \ell(m-\ell)}{(k a)^{2}} J_{\ell}^{(n-1)}(k a)\right] A_{\ell}^{j-n, P} \\
& +\left[H_{\ell}^{(n+1)}(k a)+\frac{2}{k a} H_{\ell}^{(n)}(k a)\right. \\
& \left.+\frac{n+n(n-1) \ell(m-\ell)}{(k a)^{2}} H_{\ell}^{(n-1)}(k a)\right] B_{\ell}^{j-n, P} \\
& -n_{c}^{n-1}\left[J_{\ell}^{(n+1)}\left(n_{c} k a\right)+\frac{2}{n_{c} k a} J_{\ell}^{(n)}\left(n_{c} k a\right)\right.
\end{aligned}
$$

$$
\left.\left.+\frac{n+n(n-1) \ell(m-\ell)}{\left(n_{c} k a\right)^{2}} J_{\ell}^{(n-1)}\left(n_{c} k a\right)\right] C_{\ell}^{j-n, P}\right\} .
$$

Here $J_{m}^{(n)}$ and $H_{m}^{(n)}$ denote the $n$th derivative of the Bessel functions with respect to their arguments. Because the sum over $n$ extends from 1 to $j$, the multipole terms that appear range from $A_{\ell}^{0, P}$ to $A_{\ell}^{j-1, P}$, with an identical bound for the $B_{\ell}^{j-n, P}$ and $C_{\ell}^{j-n, P}$ coefficients. Thus, as expected, the Fourier coefficients for the boundary functions depend only on solutions to problems with a lower order in $\epsilon$.

Substituting the Fourier expansions [Eqs. (23)-(25)] into Eqs. (16) and (21) we obtain the equations

$$
\begin{array}{r}
A_{m}^{j P} J_{m}(k a)+B_{m}^{j P} H_{m}(k a)-C_{m}^{j P} J_{m}\left(n_{c} k a\right)=f_{m}^{(j)}, \\
A_{m}^{j P} k J_{m}^{\prime}(k a)+B_{m}^{j P} k H_{m}^{\prime}(k a)-C_{m}^{j P} k J_{m}^{\prime}\left(n_{c} k a\right) / n_{c}=g_{m}^{(j)} .
\end{array}
$$

Eliminating the inner term $C_{m}^{j P}$, we obtain the response of the cylinder in terms of the external field and the perturbation due to the boundary:

$$
B_{m}^{j P}=\frac{\left[J_{m}^{\prime}\left(n_{c} k a\right) J_{m}(k a)-n_{c} J_{m}^{\prime}(k a) J_{m}\left(n_{c} k a\right)\right] A_{m}^{j P}-f_{m}^{(j)} J_{m}^{\prime}\left(n_{c} k a\right)+n_{c} J_{m}(k a) g_{m}^{(j)} / k}{n_{c} H_{m}^{\prime}(k a) J_{m}\left(n_{c} k a\right)-J_{m}^{\prime}\left(n_{c} k a\right) H_{m}(k a)} .
$$

This relation can be written

$$
B_{m}^{j P}=R_{m}^{P} A_{m}^{j P}+\widetilde{R}_{m}^{j P}
$$

where

$$
R_{\ell}^{P}=-\frac{n_{c} J_{m}^{\prime}(k a) J_{m}\left(n_{c} k a\right)-J_{m}^{\prime}\left(n_{c} k a\right) J_{m}(k a)}{n_{c} H_{m}^{\prime}(k a) J_{m}\left(n_{c} k a\right)-J_{m}^{\prime}\left(n_{c} k a\right) H_{m}(k a)},
$$

and

$$
\widetilde{R}_{\ell}^{j P}=\frac{-f_{m}^{(j)} J_{m}^{\prime}\left(n_{c} k a\right)+n_{c} k^{-1} J_{m}\left(n_{c} k a\right) g_{m}^{(j)}}{n_{c} H_{m}^{\prime}(k a) J_{m}\left(n_{c} k a\right)-J_{m}^{\prime}\left(n_{c} k a\right) H_{m}(k a)} .
$$

The terms $R_{m}^{P}$ are the classical "response" terms of a circular cylinder of radius $a$ to an external source. The terms $\widetilde{R}_{m}^{j P}$ ensure that the field obeys the correct condition at or$\operatorname{der} j$ on the perturbed boundary.

\section{SCATTERING FROM A FINITE SET OF CYLINDERS}

Having calculated the response of a perturbed cylinder to an arbitrary external source, we now examine how multiple scattering from the other cylinders can be incorporated. The method here can be found in various sources and is often called the multiple or multipole scattering method, ${ }^{20}$ or the Rayleigh method. ${ }^{9}$ The derivation of the method involves an integral equation that is solved by use of a Fourier ansatz on the boundary, which in turn leads to a dense complex matrix equation that is easily solved and leads to highly convergent semianalytical expressions for the fields. The derivation given here is only a sketch; more complete derivations can be found in the literature. 9,19

We recall that the field outside a given cylinder $P$ can be written [Eq. (23)]

$$
u_{j}^{e}(r, \theta)=\sum_{m=-\infty}^{\infty}\left[A_{m}^{j P} J_{m}(k r)+B_{m}^{j P} H_{m}(k r)\right] \exp (i m \theta)
$$

where, once again, $(r, \theta)$ represents the polar coordinates centered on the cylinder $P$. We now note that the singular part of this expansion describes the outgoing field from the cylinder, whereas the regular part consists of the total field contributed from the sources external to cylinder $P$. That is,

$$
\begin{aligned}
\sum_{m=-\infty}^{\infty} & A_{m}^{j P} \boldsymbol{J}_{m}(k r) \exp (i m \theta) \\
= & \text { (scattered field from all other cylinders }) \\
& +(\text { sources located at infinity }) \\
= & \sum_{Q \neq P} \sum_{m=-\infty}^{\infty} B_{m}^{j Q} H_{m}\left(k\left|\mathbf{r}-\mathbf{r}_{P Q}\right|\right) \exp \left(i m \theta_{P Q}\right) \\
& +\delta_{j, 0} \exp \left[i \mathbf{k} \cdot\left(\mathbf{r}+\mathbf{r}_{P}\right)\right]
\end{aligned}
$$

Here $\mathbf{r}_{P}$ denotes the position vector pointing from the origin to the center of cylinder $P$, and $\mathbf{r}_{P Q}=\left(r_{P Q}, \theta_{P Q}\right)$ denotes the vector pointing from the center of cylinder $Q$ to the center of cylinder $P$, as shown in Fig. 2. As an external source we have assumed an incident plane wave with 


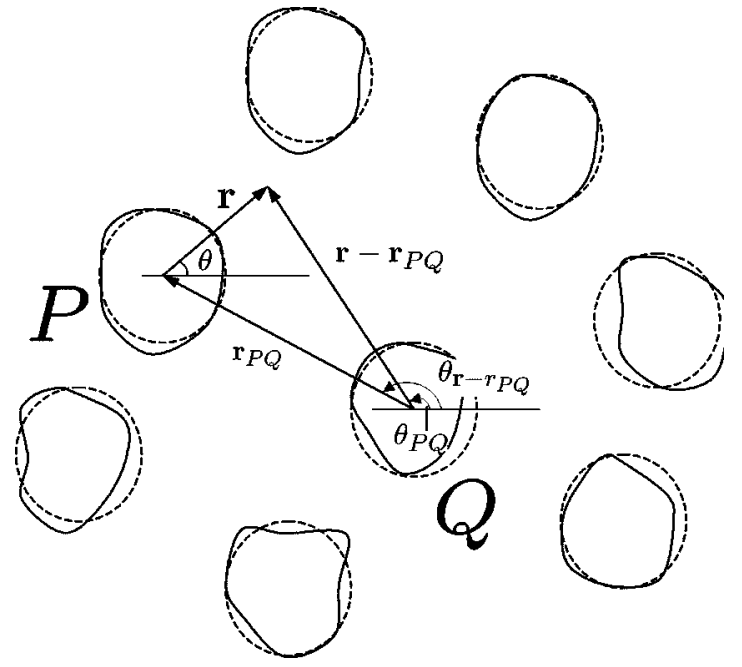

Fig. 2. Angles for the multiple-scattering case.

wave vector $\mathbf{k}=\left(k, \theta_{i}\right)$ in polar coordinates, with $\theta_{i}$ being the direction of propagation. Because the correction fields $u_{j>1}$ result from fictitious source distributions on the cylinder boundaries and not on real source distributions at infinity, the incident wave only plays a direct role for the unperturbed $(j=0)$ problem.

We now transform the fields from external sources into the coordinates of cylinder $P$. The incident plane wave can be written ${ }^{21}$

$$
\begin{aligned}
\exp \left[i \mathbf{k} \cdot\left(\mathbf{r}+\mathbf{r}_{P}\right)\right]= & \sum_{m=-\infty}^{\infty}\left[i ^ { m } \operatorname { e x p } \left(-i m \theta_{i}\right.\right. \\
& \left.\left.+i \mathbf{k} \cdot \mathbf{r}_{P}\right)\right] J_{m}(k r) \exp (i m \theta) .
\end{aligned}
$$

The Hankel function can be similarly expanded with the aid of the addition theorem for Bessel functions ${ }^{22}$ :

$$
\begin{aligned}
H_{\ell}(k \mid \mathbf{r} & \left.-\mathbf{r}_{P Q} \mid\right) \exp \left(i \ell \theta_{\mathbf{r}-\mathbf{r}_{P}}\right) \\
= & \sum_{m=-\infty}^{\infty}\left\{(-1)^{\ell-m} H_{\ell-m}\left(k r_{P Q}\right) \exp \left[i(\ell-m) \theta_{P Q}\right]\right\} \\
& \quad \times J_{m}(k r) \exp (i m \theta),
\end{aligned}
$$

where we note that always $r<r_{P Q}$; in fact, the condition $r<r_{P Q}$ puts an explicit limit on the radius of convergence of the field representation [Eq. (35)]. Substituting Eqs. (37) and (38) into Eq. (36), we obtain

$$
\begin{aligned}
\sum_{m=-\infty}^{\infty} A_{m}^{j P} J_{m}(k r) \exp (i m \theta) \\
=\sum_{m=-\infty}^{\infty}\left[\delta_{j, 0} i^{m} \exp \left(-i m \theta_{i}+i \mathbf{k} \cdot \mathbf{r}_{P}\right)\right] \times J_{m}(k r) \exp (i m \theta) \\
\quad+\sum_{m=-\infty}^{\infty} \sum_{Q \neq P} \sum_{\ell=-\infty}^{\infty}\left\{B_{\ell}^{j Q}(-1)^{\ell-m} H_{\ell-m}\left(k r_{P Q}\right)\right. \\
\left.\quad \times \exp \left[i(\ell-m) \theta_{P Q}\right]\right\} J_{m}(k r) \exp (i m \theta)
\end{aligned}
$$

Thus, equating like multipole orders, we obtain the identity

$$
A_{m}^{j P}=\delta_{j, 0} i^{m} \exp \left(-i m \theta_{i}+i \mathbf{k} \cdot \mathbf{r}_{P}\right)+\sum_{Q \neq P} \sum_{\ell=-\infty}^{\infty} \chi_{\ell-m}^{P Q} B_{\ell}^{j Q},
$$

where

$$
\chi_{m}^{P Q}=(-1)^{m} H_{m}\left(k r_{P Q}\right) \exp \left(i m \theta_{P Q}\right)
$$

are coefficients that incorporate the geometrical information relating the cylinders.

We can now incorporate the boundary condition for each order in $\epsilon$. The response of the cylinder to an arbitrary external field was already calculated in Section 4 [Eq. (32)]. If we substitute this expression into the identity that connects the fields from different cylinders [Eq. (40)], we obtain

$$
\begin{aligned}
A_{m}^{j P}= & \delta_{j, 0} i^{m} \exp \left(-i m \theta_{i}+i \mathbf{k} \cdot \mathbf{r}_{P}\right) \\
& +\sum_{Q \neq P} \sum_{\ell=-\infty}^{\infty} \chi_{\ell-m}^{P Q}\left(R_{\ell}^{Q} A_{\ell}^{j Q}+\widetilde{R}_{\ell}^{j Q}\right) .
\end{aligned}
$$

This can then be rearranged to form the infinite linear system

$$
\begin{aligned}
A_{m}^{j P}-\sum_{Q \neq P} \sum_{\ell=-\infty}^{\infty} \chi_{\ell-m}^{P Q} R_{\ell}^{Q} A_{\ell}^{j Q}= & \delta_{j, 0} i^{m} \exp \left(-i m \theta_{i}+i \mathbf{k} \cdot \mathbf{r}_{P}\right) \\
& +\sum_{Q \neq P} \sum_{\ell=-\infty}^{\infty} \chi_{\ell-m}^{P Q} \widetilde{R}_{\ell}^{j Q} .
\end{aligned}
$$

Equation (43) is a dense complex linear system that is readily solved. To accomplish this, one probably should write the system in block matrix form, with each block containing a set of multipole coefficients for a given cylinder. For the $j$ th order, we define the $P$ th blocks of the column matrices $\mathbf{A}^{(j)}, \mathbf{I}_{\text {inc }}^{(j)}$, and $\widetilde{\mathcal{R}}^{(j)}$ as

$$
\begin{aligned}
& {\left[\mathbf{A}^{(j)}\right]_{m, P}=A_{m}^{j P},} \\
& {\left[\mathbf{I}_{\text {inc }}^{(j)}\right]_{m, P}=\delta_{j, 0} \delta_{\ell, m} i^{m} \exp \left(-i m \theta_{i}+i \mathbf{k} \cdot \mathbf{r}_{P}\right),} \\
& {\left[\widetilde{\mathcal{R}}^{(j)}\right]_{m, P}=\sum_{Q \neq P} \sum_{\ell=-\infty}^{\infty} \chi_{\ell-m}^{P Q} \widetilde{R}_{\ell}^{j Q},}
\end{aligned}
$$

and the elements of the $(P, Q)$ th block of a block matrix $\mathcal{R}$ can then be defined as

$$
[\mathcal{R}]_{\ell, m, P, Q}=\delta_{l, m} \delta_{P, Q}-\chi_{\ell-m}^{P Q} R_{\ell}^{Q}
$$

The linear system [Eq. (43)] can then be written as the matrix equation

$$
\mathcal{R} \mathbf{A}^{(j)}=\mathbf{I}_{\mathrm{inc}}{ }^{(j)}+\widetilde{\mathcal{R}}^{(j)} .
$$

It is interesting to note that the matrix $\mathcal{R}$ does not depend on the character of the perturbation. This can be seen from the definition of the terms $R_{\ell}^{Q}$ in Eq. (33). The information from the boundary perturbation function $h(\theta)$ is contained in the driving term $\widetilde{\mathcal{R}}^{(j)}$ on the right-hand side, which also depends on the multipole coefficients arising from solutions of lower order in $\epsilon$. This means that the system [Eq. (48)] must be inverted only once in order to 
find the solution to successive orders in $\epsilon$. In addition, the effect of any number of different perturbations can be computed simply through recalculation of the components of $\widetilde{\mathcal{R}}^{(j)}$. This gives a significant computational advantage for evaluation of large structures such as photonic crystals. The matrix inverse depends only on the unperturbed problem; once this is solved, it is a simple matter of back substitution to find the solution to any higher order and for any weak perturbation.

\section{NORMALIZATION AND CONVERGENCE OF THE SYSTEM}

We now give a few words as to the properties of the system [Eq. (43)]. If one tries to directly solve this system, one quickly runs into numerical problems due to bad conditioning, and this warrants a short discussion as to how these difficulties can be overcome. The work presented in this section can be found in the literature, albeit in scattered form. ${ }^{23,19}$

First we note that the above system can be put in a slightly more convenient form by replacing the definition [Eq. (32)] with

$$
A_{m}^{j P}=\frac{1}{R_{m}^{P}} B_{m}^{j P}-\frac{\widetilde{R}_{m}^{j P}}{R_{m}^{P}}=: M_{m}^{P} B_{m}^{j P}-\tilde{M}_{m}^{P} .
$$

Substituting this expression into Eq. (42) results in the system

$$
\begin{aligned}
B_{m}^{j P}-\sum_{Q \neq P} \sum_{\ell} \frac{\chi_{\ell-m}^{P Q}}{M_{m}^{P}} B_{\ell}^{j Q}= & \frac{1}{M_{m}^{P}}\left\{\delta_{j, 0} i^{m} \exp \left(-i m \theta_{i}+i \mathbf{k} \cdot \mathbf{r}_{P}\right)\right. \\
& \left.+\tilde{M}_{m}^{P}\right\},
\end{aligned}
$$

which is more compact.

Both systems [Eqs. (43) and (50)] are badly conditioned in the limit that the number of multipole orders becomes large. To see this we note ${ }^{22}$ that in the limit of large $m$,

$$
\begin{aligned}
\chi_{m}^{P Q} & =(-1)^{m} H_{m}\left(k r_{P Q}\right) \exp \left(i m \theta_{P Q}\right) \\
& \sim \exp \left[i\left(m \theta_{P Q}+\pi\right)\right]\left(\frac{2}{\pi m}\right)^{1 / 2}\left(\frac{e k r_{P Q}}{2 m}\right)^{-|m|} \\
& \sim \mathcal{O}\left(m^{|m|-1 / 2}\right) .
\end{aligned}
$$

As $m \rightarrow \infty$ the terms $\tilde{M}_{m}^{P}$ are of the order

$$
M_{m}^{P}=\left(R_{m}^{P}\right)^{-1} \sim-\frac{i\left(\frac{2}{\pi m}\right)^{1 / 2}\left(\frac{e k a}{2 m}\right)^{-|m|}}{\frac{1}{(2 \pi m)^{1 / 2}}\left(\frac{e k a}{2 m}\right)^{|m|}} \mathcal{O}\left(m^{+2|m|}\right) .
$$

If we fix $m$ and let $\ell \rightarrow \infty$, the off-diagonal terms in the linear system [Eq. (50)] then are

$$
\frac{\chi_{\ell-m}^{P Q}}{\tilde{M}_{m}^{P}} \sim \mathcal{O}\left(\ell^{+|\ell|-1 / 2}\right) \text {. }
$$

On the other hand, if we fix $\ell$ as $m \rightarrow \infty$, we find that

$$
\frac{\chi_{\ell-m}^{P Q}}{\tilde{M}_{m}^{P}} \sim \mathcal{O}\left(m^{-|m|-1 / 2}\right) .
$$

This behavior for large $\ell$ and $m$ results in a matrix that is extremely difficult to invert and will cause problems when a high accuracy is desirable. To overcome this difficulty we can define a new vector of unknowns

$$
X_{m}^{P}=\left(\left|R_{m}^{P}\right|\right)^{1 / 2} B_{m}^{j P} .
$$

Substitution into Eq. (50) then leads to the system

$$
\begin{aligned}
X_{m}^{P}-\sum_{Q \neq P} \sum_{\ell} \frac{\chi_{\ell-m}^{P Q}}{\left(\left|M_{m}^{P} M_{\ell}^{Q}\right|\right)^{1 / 2}} X_{\ell}^{Q}= & \frac{1}{\left(\left|M_{m}^{P}\right|\right)^{1 / 2}}\left\{\delta _ { j , 0 } i ^ { m } \operatorname { e x p } \left(-i m \theta_{i}\right.\right. \\
& \left.\left.+i \mathbf{k} \cdot \mathbf{r}_{P}\right)+\tilde{M}_{m}^{P}\right\} .
\end{aligned}
$$

In this case, as we hold $m$ constant and let $\ell \rightarrow \infty$, the offdiagonal terms are

$$
\frac{\chi_{\ell-m}^{P Q}}{\left(\left|M_{m}^{P} M_{\ell}^{Q}\right|\right)^{1 / 2}} \sim \mathcal{O}\left(\ell^{-2|\ell|-1 / 2}\right) .
$$

When we hold $\ell$ constant and let $m \rightarrow \infty$, we obtain

$$
\frac{\chi_{\ell-m}^{P Q}}{\left(\left|M_{m}^{P} M_{\ell}^{Q}\right|\right)^{1 / 2}} \sim \mathcal{O}\left(m^{-2|m|-1 / 2}\right) .
$$

Thus the new system has elements that decay with order $\mathcal{O}\left(m^{-2|m|}\right)$ away from the main diagonal. This means that the multipole coefficients $X_{m}^{P}$ will decay similarly rapidly and therefore that the coefficients $B_{m}^{j P}$ will decay with or$\operatorname{der} \mathcal{O}\left(m^{-|m|}\right)$.

\section{RESULTS OF SIMULATIONS}

We now present results obtained from solving the system of Eq. (56) for various types of perturbation. The system is truncated so as to include a given number of multipole orders, and this results in a matrix equation for the vector of unknowns $X_{m}^{P}$. As a first example we consider two cylinders consisting of air holes in InP, which has a refractive index of 3.17. The perturbation function $h(\theta)$ consists of a sum of randomly generated Fourier coefficients [as in Eq. (26)] with the coefficients $h_{m}^{(1)}$ chosen such that $h_{m}^{(1)}$ $=0$ for $|m|>5$. The perturbation function is then band limited and has a coherence length (taken around the circumference of the unperturbed cylinder) of $L_{c}=2 \pi a / 5$, where $a$ is the unperturbed radius of the cylinders, chosen in this case so that $a=0.186 \mu \mathrm{m}$. One must take care that the crinkliness of the boundary (and of its derivatives with respect to $\theta$ ) can be resolved by the final field. To this end we choose a maximum of 25 multipole orders. Figure 3 shows the $z$ component of the magnetic field for the TE polarization at a vacuum wavelength of $\lambda=1.55 \mu \mathrm{m}$.

The error in the depiction of the fields in this problem can be measured by observing how well the field matches the boundary conditions in Eqs. (5) and (6) on the perturbed boundary. Figure 4 depicts the maximum error on the left-most cylinder in Fig. 3 obtained for different asymptotic orders [i.e., the maximum value of $j$ in Eq. (7)] ranging from 1 to 5 , with the error being expressed as a percentage of the magnitude of the incident field. It can 


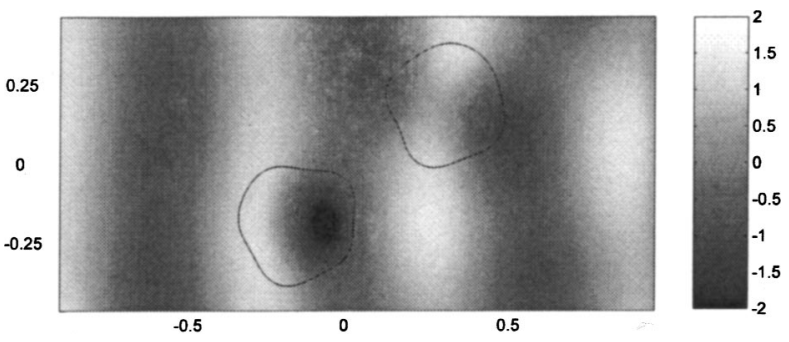

Fig. 3. Real part of the $z$ component of the magnetic field for two dielectric cylinders with sidewall perturbations. The incident wave is from the left, with wavelength $1.55 \mu \mathrm{m}$.

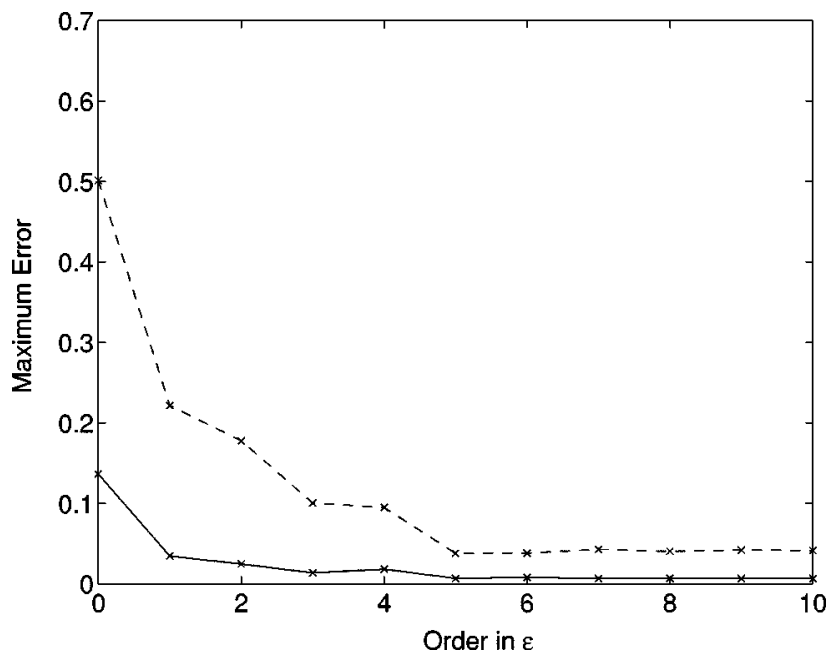

Fig. 4. Maximum error, measured on the boundary of the lefthand cylinder in Fig. 3, in the field value boundary condition (solid curve) and the derivative boundary condition (dashed curve) expressed as a percentage of the magnitude of the incident field.

be seen that the solution approaches a small error very quickly before being limited by the accuracy of the evaluation of the terms in the system [Eq. (56)].

In Figs. 5-8 we present results for a photonic crystal waveguide bend consisting of 76 cylinders. The structure consists of air holes in an InP background, arranged in a hexagonal lattice with radius $a=186 \mathrm{~nm}$ and period $d$ $=484 \mathrm{~nm}$. The photonic crystal possesses a band gap with a range of 158-247 THz. Because most of the calculation time is taken up in the evaluation and inversion of the matrix $\mathcal{R}$ in Eq. (48), and because this quantity does not depend on the particular perturbation being studied, a number of different perturbations of the boundary can be evaluated with little extra computational cost. We present four variations; Fig. 5 shows the unperturbed problem for circular cylinders. In this case the power transmission around the bend is measured to be $80 \%$, or $-0.97 \mathrm{~dB}$. Figure 6 shows a "rounded triangle" perturbation consisting of randomly oriented quasi-triangular holes. The power transmission in this case is reduced to $62 \%$, or $-2.0 \mathrm{~dB}$. Figure 7 shows the same waveguide in an array of quasiellipses [for which $h(\theta)=\sqrt{2} \cos 2 \theta$ ], exhibiting a transmission of $77 \%$, or $-1.1 \mathrm{~dB}$. Finally, in Fig. 8 we show a photonic crystal with randomly perturbed sidewalls, such that $h_{|m|>5}^{(1)}=0$ in Eq. (26). In this configuration the transmission is slightly improved to $82 \%$, or $-0.87 \mathrm{~dB}$. In each

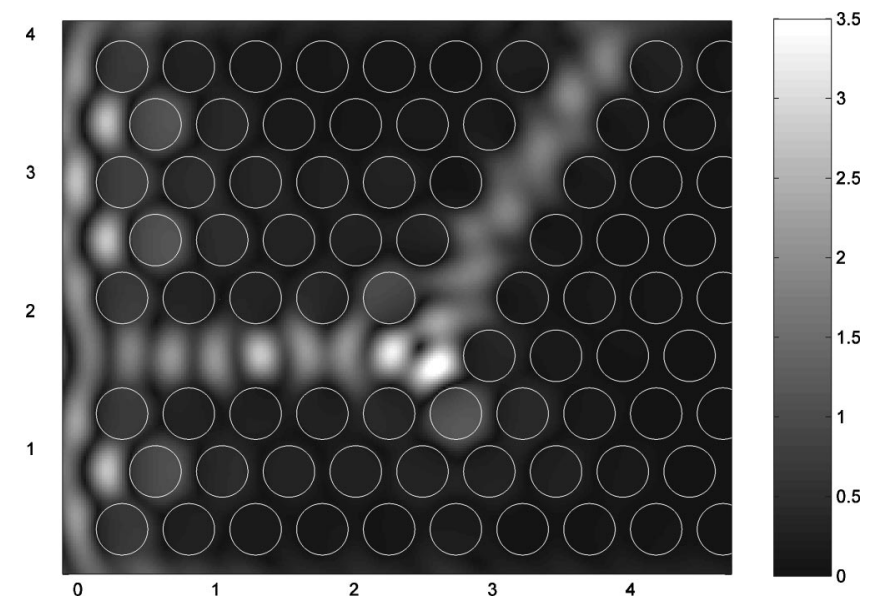

Fig. 5. Magnitude of the $z$ component of the magnetic field for a photonic crystal waveguide bend consisting of voids in InP. The radius of the voids is $186 \mathrm{~nm}$; the lattice spacing is $484 \mathrm{~nm}$. The incident wave is from the left, with wavelength $1.55 \mu \mathrm{m}$. The power transmission around the bend is $80 \%$, or $-0.97 \mathrm{~dB}$. The dimensions given here, and in all of the following figures, are in micrometers.

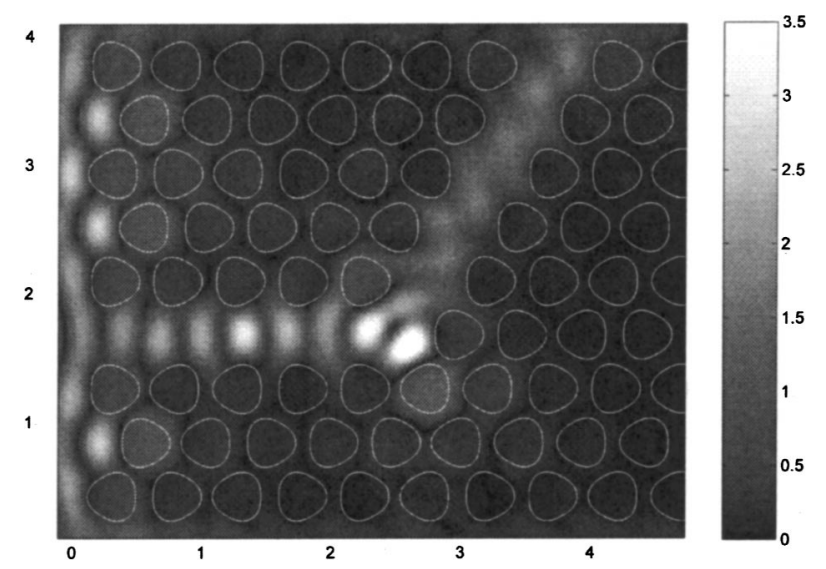

Fig. 6. Magnitude of the $z$ component of the magnetic field for a photonic crystal waveguide bend with rounded triangle perturbation. The rms size of the perturbation is $18.6 \mathrm{~nm}$, or $10 \%$ of the cylinder radius. The power transmission around the bend is $62 \%$, or $-2.0 \mathrm{~dB}$.

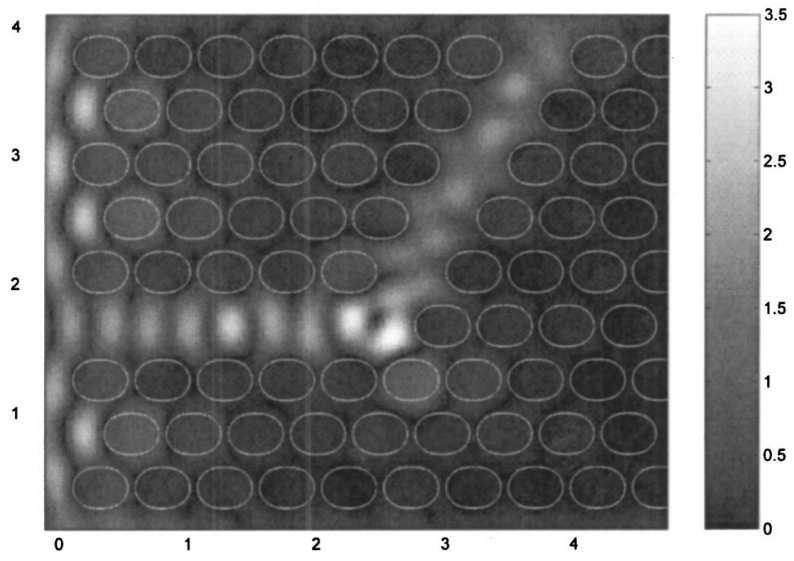

Fig. 7. Magnitude of the $z$ component of the magnetic field for a photonic crystal waveguide bend constructed out of quasiellipses, for which $h(\theta)=\sqrt{2} \cos 2 \theta$. The rms size of the perturbation is $18.6 \mathrm{~nm}$, or $10 \%$ of the cylinder radius. The power transmission around the bend is $77 \%$, or $-1.1 \mathrm{~dB}$. 


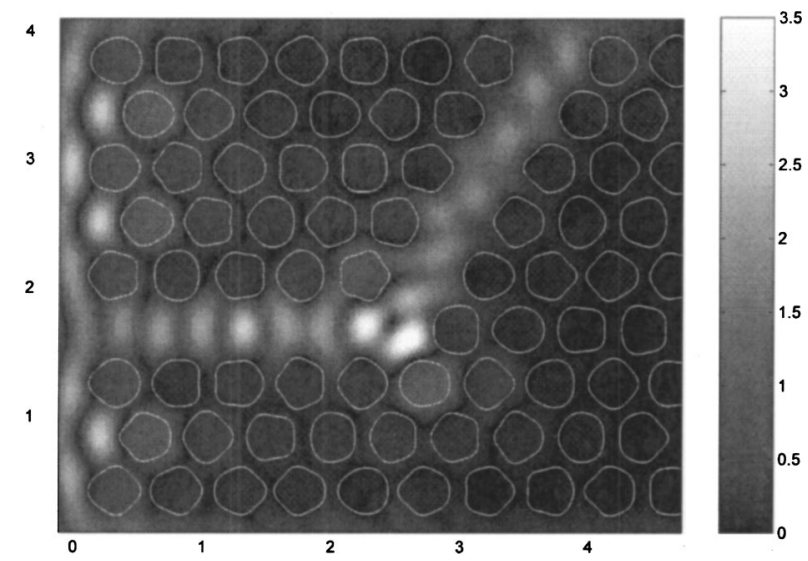

Fig. 8. Magnitude of the $z$ component of the magnetic field for a photonic crystal waveguide bend with random sidewall perturbations. The rms size of the perturbation is $18.6 \mathrm{~nm}$, and the void boundaries possess an angular coherence length of 1.25 radians. The power transmission around the bend is $82 \%$, or $-0.87 \mathrm{~dB}$.

(a)

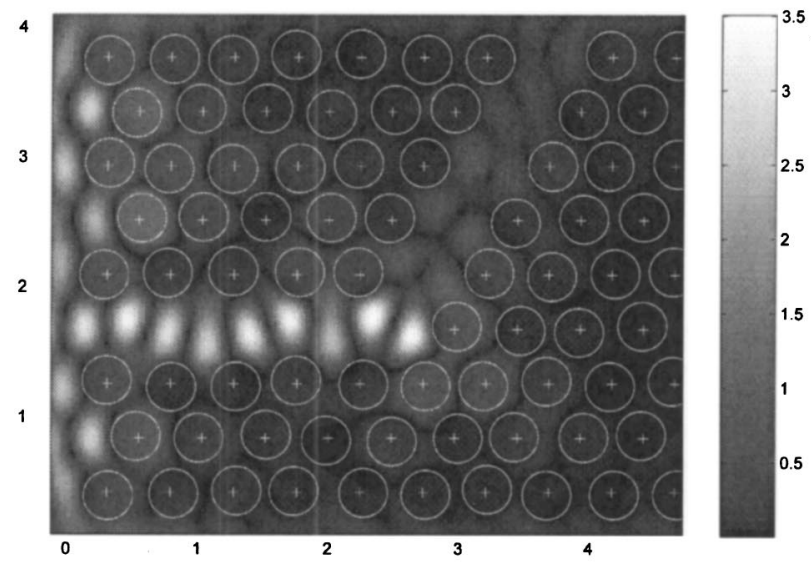

(b)

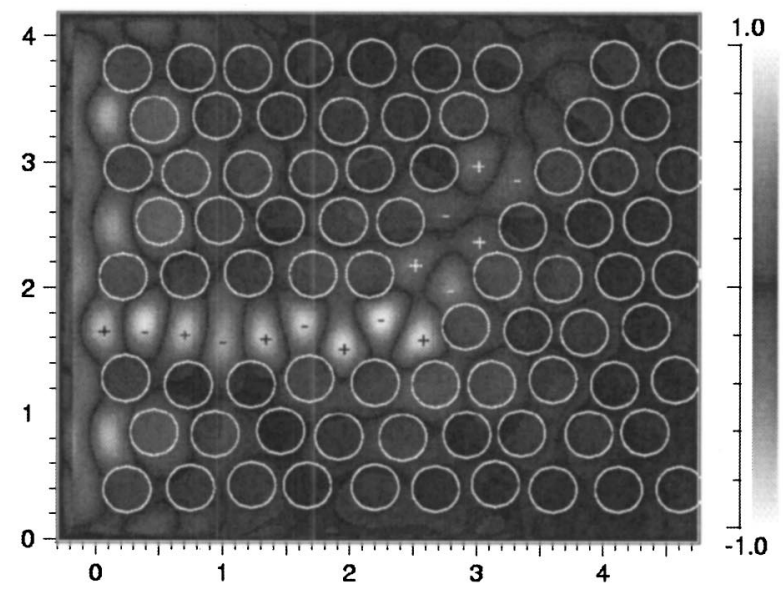

Fig. 9. (a) Magnitude of the $z$ component of the magnetic field for a photonic crystal waveguide bend with random positional disorder. The crosses show the centers of the original, unperturbed cylinders. It is observed that although the disorder is weak the transmission can be reduced considerably-in this case the power transmission around the bend is $1.5 \%$, or $-18 \mathrm{~dB}$. (b) Calculation for the same structure by use of a commercial 2D FDTD software package. Plotted is the dominant field component taken at the instant when the field in the input waveguide is at maximum. The plus and minus signs show the changing sign of the field.

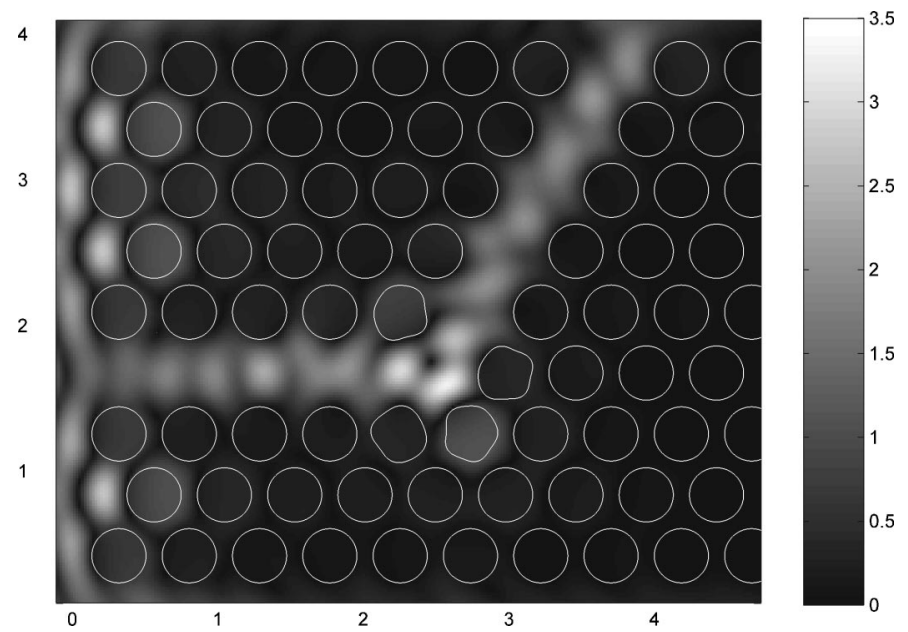

Fig. 10. Magnitude of the $z$ component of the magnetic field for a photonic crystal waveguide bend with perturbations around the corner of the bend. The power transmission around the bend is $94 \%$, or $-0.26 \mathrm{~dB}$, significantly greater than that of the unperturbed waveguide.

case the rms perturbation is $\epsilon=18.6 \mathrm{~nm}$, or $10 \%$ of the cylinder radius.

It is observed that, although the transmission may vary, the integrity of the bandgap remains intact for each perturbation. This is perhaps to be expected, because the frequency lies more or less in the middle of the bandgap, which is quite wide ( $89 \mathrm{THz}$ for the filling fraction here). It can be seen from Fig. 5 that the dominant mechanism for the transmission is a corner resonance, which may be disrupted (or enhanced) by the sidewall deformations.

We can add a weak positional disorder by keeping $h_{m \neq 1}^{(1)}=0$ in Eq. (26) and choosing $h_{ \pm 1}^{(1)}$ randomly. This retains the circular shape of the cylinders while displacing each by an amount $\epsilon=9.3 \mathrm{~nm}$ in a random direction. In Fig. 9(a) we see that this can result in almost zero transmission; the power transmission is in this case measured to be $1.5 \%$, or $-18 \mathrm{~dB}$. In this configuration the corner resonance is disrupted in such a way as to couple to the second-order waveguide mode, which is below cutoff at this frequency.

As an additional check on the validity of the model, we have compared it with results from a commercial FDTD software package (RSoft FullWAVE). For this we chose an identical structure to that shown in Fig. 9(a), i.e., that of the disordered waveguide bend. This structure was easy to implement by use of commercial computer-aided design software and also represents the most dramatic influence of disorder on the transmission. It can be seen in Fig. 9 that the field structure is not significantly different with the two methods. In addition, the transmission suppression due to the disorder (i.e., the transmission for the disordered structure divided by the transmission for the unperturbed structure) is calculated to be $98.13 \%$ (17.3 dB) with the FDTD software, and $98.08 \%(17.2 \mathrm{~dB})$ with the new method, a difference that is well within the error margin of the software.

Finally, we note that the shape of the elements of the photonic crystal can be "tuned" in order to achieve increased transmission. In Fig. 10 we have randomly modified the shapes of the four cylinders around the wave- 
guide bend and chosen one configuration that exhibits a higher transmission than that of the unperturbed bend. In this manner we are able to achieve a transmission of $94 \%$, or $-0.26 \mathrm{~dB}$, which is considerably greater than that of the unperturbed structure. It is anticipated that there is ample scope for the use of this method to optimize the shapes of the individual elements within photonic crystals.

\section{CONCLUSION}

We have used a combination of asymptotic and multipole methods to calculate the effect of sidewall perturbations on scattering from groups of cylinders. This method combines accuracy with a small computational load so that many configurations can be studied once the solution of the original unperturbed problem is known. Such a method would make a valuable tool for the study of sidewall perturbation on photonic-crystal waveguides and could be used to optimize the shapes of cylinders in photonic-crystal bends, Y junctions, and other structures. The method can also be used to study the effects of weak disorder and localization in photonic crystals.

\section{ACKNOWLEDGEMENTS}

This work was supported by the Deutsche Forschungsgemeinschaft (DFG) through the Center for Functional Nanostructures within project A3.1. C. G. Poulton acknowledges helpful comments and suggestions from A. B. Movchan, University of Liverpool, and S. Guenneau, University College, London. The authors thank the referees for a careful and constructive reading of the text.

\section{REFERENCES}

1. C. J. M. Smith, H. Benisty, S. Olivier, M. Rattier, C. Weisbuch, T. F. Krauss, R. M. De La Rue, R. Houdré, and U. Oesterle, "Low-loss channel waveguides with twodimensional photonic crystal boundaries," Appl. Phys. Lett. 77, 2813-2815 (2000).

2. J. Zimmermann, M. Kamp, R. Schwertberger, J. Reithmaier, A. Forchel, and R. März, "Efficient light transmission through InP-based photonic crystal waveguides," Electron. Lett. 38, 178-179 (2002).

3. S. J. McNab, N. Moll, and Y. A. Vlasov, "Ultra-low loss photonic integrated circuit with membrane-type photonic crystal waveguides," Opt. Express 11, 2827-2939 (2003), http://www.opticsexpress.org

4. O. C. Zienkiewicz and R. L. Taylor, The Finite Element Method (McGraw-Hill Education, London, 1989).

5. M. Fujii and W. J. R. Hoefer, "Application of waveletGalerkin method to electrically large optical waveguide problems," IEEE MTT-S Int. Microwave Symp. Dig. TU3E3, 239-242 (2000).
6. J. R. Wait, Electromagnetic Radiation from Cylindrical Structures (Pergamon, London, 1959), pp. 149-159.

7. S. Agmon, A. Douglis, and L. Nirenberg, "Estimates near the boundary for solutions of elliptic partial differential equations satisfying general boundary conditions," Commun. Pure Appl. Math. 12, 623-727 (1959).

8. V. V. Jikov, S. M. Kozlov, and O. A. Oleinik, Homogenization of Differential Operators and Integral Functionals (Springer, Berlin, 1994).

9. R. C. McPhedran, N. A. Nicorovici, L. C. Botten, and K.-D. Bao, Green's Function, Lattice Sums and Rayleigh's Identity for a Dynamic Scattering Problem, Vol. 96 of IMA Volumes in Mathematics and its Applications (SpringerVerlag, New York, 1997), pp. 155-180.

10. S. Fan, P. R. Villeneuve, and J. D. Joannopolous, "Theoretical investigations of fabrication-related disorder on the properties of photonic crystals," J. Appl. Phys. 78, 1415-1418 (1995).

11. A. Chutinan and S. Noda, "Effects of structural fluctuations on the photonic bandgap during fabrication of a photonic crystal,” J. Opt. Soc. Am. B 16, 240-244 (1999).

12. Z. Y. Li, X. D. Zhang, and Z. Q. Zhang, "Disordered photonic crystals understood by a perturbation formalism," Phys. Rev. B 61, 15738-15747 (2000).

13. Z. Y. Li and Z. Q. Zhang, "Fragility of photonic band gaps in inverse-opal photonic crystals," Phys. Rev. B 62, 1516-1519 (2000)

14. M. A. Kaliteevski, J. M. Martinez, D. Cassagne, and J. P. Albert, "Disorder-induced modification of the transmission of light in a two-dimensional photonic crystal," Phys. Rev. B 66, 113101-113101-4 (2002).

15. A. A. Asatryan, P. A. Robinson, L. C. Botten, R. C. McPhedran, N. A. Nicorovici, and C. M. de Sterke, "Effects of disorder on wave propagation in two-dimensional photonic crystals," Phys. Rev. E 60, 6118-6127 (1999).

16. C. G. Poulton, S. Guenneau, A. B. Movchan, and A. Nicolet, "Transverse propagating waves in perturbed periodic structures," in International Union of Theoretical and Applied Mechanics Proceedings (Kluwer, Dordrecht, The Netherlands, 2002), pp. 147-157.

17. M. H. Lean, "Novel integration for scattering from multilayered dielectric cylinders of arbitrary cross section," IEEE Trans. Magn. 20, 1476-1479 (2004).

18. Y. I. Tanaka, T. Asano, Y. Akahane, B. S. Song, and S. Noda, "Theoretical investigation of a two-dimensional photonic crystal slab with truncated cone air holes," Appl. Phys. Lett. 82, 1661-1663 (2003).

19. T. P. White, B. T. Kuhlmey, R. C. McPhedran, D. Maystre, G. Renversez, C. M. de Sterke, and L. C. Botten, "Multipole method for microstructured optical fibers. I. Formulation," J. Opt. Soc. Am. B 19, 2322-2330 (2002).

20. V. Rokhlin, "Rapid solution of integral equations of scattering theory in two dimensions," J. Comput. Phys. 86, 414-439 (1990).

21. G. N. Watson, A Treatise on the Theory of Bessel Functions (Cambridge University Press, Cambridge, UK, 1980).

22. M. Abramowitz and I. A. Stegun, eds., Handbook of Mathematical Functions (Dover, New York, 1972).

23. C. G. Poulton, A. B. Movchan, R. C. McPhedran, N. A. Nicorovici, and Y. A. Antipov, "Eigenvalue problems for doubly periodic elastic structures and phononic band gaps," Proc. R. Soc. London, Ser. A 456, 2543-2559 (2000). 